\title{
Identification of a Peptide from In vivo Bacteriophage Display with Homology to EGFL6: A Candidate Tumor Vasculature Ligand in Breast Cancer
}

\author{
Benjamin M Larimer ${ }^{1,2 *}$ and Susan L Deutscher ${ }^{1,2}$ \\ ${ }^{1}$ Research Service, Harry S. Truman Veterans Memorial Hospital, Columbia, MO, USA \\ ${ }^{2}$ Department of Biochemistry, University of Missouri, Columbia, MO, USA
}

\begin{abstract}
Background: A crucial step in tumorigenesis is the recruitment of novel vasculature to the site of neoplasia. Currently, a number of high throughput techniques are employed to identify genes, mRNA and proteins that are aberrantly expressed in tumor vasculature. One drawback of such techniques is the lack of functional in vivo data that they provide. Bacteriophage (phage) display has been demonstrated in vivo to select peptides that home to tumors and tumor vasculature. The peptides can be compared to sequences of putative cancer-related proteins, in order to identify novel proteins essential for tumorigenesis.
\end{abstract}

Objectives: It was hypothesized that an in vivo selection for phage which targeted human breast cancer xenografts could identify peptides with homology to cancer-related proteins for in vivo imaging of breast cancer.

Methods: Following four rounds of in vivo selection in human MDA-MB-435 breast cancer xenografted mice, peptide 3-G03 was discovered with significant homology to a putative secreted protein termed EGFL6. Egfl6 mRNA is upregulated in several transcriptomic analyses of human cancer biopsies, and the protein may play a role in tumor vascularization.

Results: Egfl6 mRNA expression was demonstrated in MDA-MB-435 cells and EGFL6 protein was secreted from these cells. Based on homology of 3-G03 to EGFL6, an EGFL6 peptide was synthesized and shown to target MDA-MB-435 cells. EGFL6 peptide was radiolabeled with ${ }^{111} \mathrm{In}$ and analyzed for biodistribution and tumor imaging capabilities. Single photon emission computed tomography imaging revealed uptake of the peptide in a manner consistent with other tumor vasculature targeting agents.

Keywords: Phage display; Molecular imaging; Peptides; EGFL6; Breast cancer; Tumor vasculature; SPECT/CT

Abbreviations: Egfl6: Epidermal Like Growth Factor-6; EGF: Epi-
dermal Growth Factor; SCID: Severe Combined Immunodeficiency;
Chaps: 3-[(3-Cholamidopropyl) Dimethylammonio]-1-Propanesul-
fonate; TBST: Tris Buffered Saline and Tween; DOTA: 1,4,7,10-Tet-
raazacyclododecane-1,4,7,10-Tetraacetic Acid

\section{Introduction}

Much effort has been placed on identifying the process of human tumor vascularization [1]. As tumor size increases, new vasculature is required to provide blood flow and nutrients to the growing malignancy, a process termed angiogenesis [2]. The network of blood vessels in each organ, including tumors, is differentiated by tissue specific expression of ligands and receptors [3]. These proteins serve as a molecular address, and in the case of tumorigenesis, may prove effective for delivery of imaging agents or cytotoxic drugs [4]. Although tumor vasculature proteins such as vascular endothelial growth factor, $\alpha_{v} \beta_{3}$ integrin, and platelet derived growth factor are well known, resistance to therapies targeting these proteins reveals that tumor vasculature is a complex system that remains incompletely characterized [5]. The ability to identify and help validate novel ligands and their corresponding receptors responsible for tumor angiogenesis would prove advantageous. Not only could the ligands serve as potential targeting vectors for imaging applications, but blockade of receptors could prevent increased blood supply for a tumor and limit its growth.

In order to determine novel cancer biomarkers, techniques must be used that illuminate cancer-specific ligands and receptors. Deciphering the differential protein expression between malignant and non- malignant cells is often attempted using high throughput screening methods, due to the complex nature of the tumor microenvironment. One process for identifying novel cancer-specific ligands and receptors is mRNA profiling, which has been used to identify genes with significantly higher transcription levels in tumors [6-8]. Human cancer transcriptional profiles have served to create a reservoir of hypothetical tumor interacting transcripts, encoding for tumor vasculature proteins such as adlican, collagen type-XI alpha-1, glycoprotein M6B and epidermal growth factor-like domain multiple 6 (egfl6) [9]. The egfl6 transcript, in particular, was first reported in several fetal tissues and human glioma tumor biopsy samples using a high throughput screening by hybridization technique [10]. Recently, several human tumor biopsy transcription analyses have indicated egfl6 mRNA is expressed at high levels in meningioma, glioma and ovarian and breast carcinomas, while levels in normal tissues were virtually undetectable [6,8,11-13]. The reports of tumor specific egfl6 expression suggest a need for investigation into its possible role in tumorigenesis.

*Corresponding author: Benjamin Michael Larimer, Department of Biochemistry University of Missouri, 117 Schweitzer Hall, Columbia, MO 65211, USA, Tel: 573882-1493; Fax: 573-884-4597; E-mail: Bmlyr4@mail.missouri.edu

Received March 03, 2014; Accepted March 24, 2014; Published April 01, 2014

Citation: Larimer BM, Deutscher SL (2014) Identification of a Peptide from In vivo Bacteriophage Display with Homology to EGFL6: A Candidate Tumor Vasculature Ligand in Breast Cancer. J Mol Biomark Diagn 5: 178. doi:10.4172/21559929.1000178

Copyright: (C) 2014 Larimer BM, et al. This is an open-access article distributed under the terms of the Creative Commons Attribution License, which permits unrestricted use, distribution, and reproduction in any medium, provided the original author and source are credited 
The egfl6 gene encodes for an approximately $60 \mathrm{kDa}$ secreted protein with Epidermal Growth Factor (EGF) structural homology [14]. Although EGFL6 has been detected at the mRNA level in numerous cancers, the protein has not been detected in carcinoma cells and little is understood regarding its in vivo function. In vitro, full-length recombinant EGFL6 has been demonstrated to induce migration and angiogenesis in endothelial cells through activation of the extracellular signal-related kinase pathway [14]. These data suggest that EGFL6 may contribute to vascularization of new and perhaps malignant tissue. However, its roles in both development and tumorigenesis remain unclear.

One method of exploring the vast array of protein interactions and associations in a system such as the tumor vasculature is through bacteriophage (phage) display [15]. A single phage library can contain up to $10^{9}$ unique peptide sequences, offering a sizeable potential for selection of a peptide fragment of a natural ligand, such as EGFL6, which is thought to be specific to tumor vasculature [16]. Previous selections have demonstrated the feasibility of phage-based ligand identification, most notably isolating the peptide RGD [17]. The RGD motif was identified in 28 of 32 phage-displayed peptides selected for binding to $\alpha_{5} \beta_{1}$ integrin, and consequently demonstrated to have high affinity for a number of integrins, including $\alpha_{v} \beta_{3}[17,18]$. Use of peptide phage display to identify binding epitopes, such as RGD, in vitro has spawned investigation into the ability of phage display to isolate tissue and tumor specific peptides in vivo [19]. In vivo phage display has previously identified tumor vasculature-homing peptides, and specific tripeptide sequences have been mapped to the vasculature of numerous human organs [20]. Additionally, our laboratory has developed a strategy for isolating not only tumor vasculature but also solid tumor-specific peptides [21]. These works indicate that phage can localize specifically to tumors, and recovered phage can be used to identify receptor-binding peptide epitopes.

It was hypothesized that in vivo phage display could be used to select peptides which mimic tumor-associated ligands. The peptides would serve not only as tumor imaging vectors but may also be used to help validate novel tumor biomarkers. Phage displayed peptides with homology to a known protein could help validate potential ligands identified by genomic or transcriptomic studies, or elucidate possible proteins underrepresented or absent from traditional proteomic analyses. To test this idea, a phage library was subjected to four rounds of in vivo selection in mice bearing human MDA-MB-435 breast cancer xenografts. Displayed peptides of phages recovered from the tumors were analyzed by the Basic Local Alignment Search Tool (BLAST). Although a number of peptides matched potential tumor related proteins, one peptide, with 9 of 14 amino acids identical (GTKSKCCYSLRRSS versus GTKLACCYGWRRNS) to EGFL6, was chosen for further study due to its significant homology and the growing evidence that EGFL6 is a potential tumor vasculature ligand. The tumor cell line used for selection, as well as several other cancer and non-cancer cell lines, was probed for mRNA and protein expression of EGFL6. Additionally, the tumor targeting and SPECT imaging properties of the EGFL6 peptide were investigated in vivo.

\section{Materials and Methods}

\section{Materials}

Materials for cell culture were purchased from Invitrogen (Carlsbad, CA). Unless otherwise specified, all reagents and materials were obtained from Sigma Chemical Co. (St. Louis, MO).

\section{Mouse strains and handling}

Four- to 6-week-old Severe Combined Immunodeficient (SCID) out bred mice (Taconic, Germantown, NY) was maintained in approved pathogen-free institutional housing. Animal studies were conducted as outlined in the NIH Guidelines for the Care and Use of Laboratory Animals and the Policy and Procedures for Animal Research of the Harry S. Truman Veterans Memorial Hospital. MDAMB-435 human breast cancer xenografts $\left(5 \times 10^{6}\right)$ were established by subcutaneous injection into the flank of SCID mice. Mice were utilized when visual tumors $\left(\sim 1-3 \mathrm{~mm}^{3}\right)$ formed after approximately 5 weeks.

\section{Phage display selection and analysis}

In vivo phage display was performed as described previously [21] Briefly, a phage library containing approximately $1 \times 10^{12}$ tetracycline transducing units of phage was injected into SCID mice bearing MDAMB-435 human breast cancer xenografts. Phages were allowed to circulate for $1 \mathrm{~h}$ and following perfusion with Phosphate Buffered Saline (PBS), tumors were excised and frozen in liquid nitrogen. Tumors were manually homogenized and bound phages eluted by incubation with $2.5 \% \quad(\mathrm{w}: \mathrm{v}) \quad 3$-[(3-cholamidopropyl) dimethylammonio]-1propanesulfonate (CHAPS) solution. Recovered phages were used to infect $\log$ phase K91BK E. coli cells, amplified for $16 \mathrm{~h}$ in a $37^{\circ} \mathrm{C}$ incubator with shaking and purified by polyethylene glycol/sodium chloride precipitation [22]. Purified phages were quantified and used for subsequent rounds of selection, for a total of four selection rounds. Following the final round of selection, individual phages were isolated and their relevant DNA was sequenced in order to ascertain the displayed foreign peptide of each phage. Peptide sequences were then queried using the BLAST search program for sequence homology to proteins with known or putative cancer correlations [23].

\section{EGFL6 RT-PCR}

In order to assay egfl6 mRNA expression, three human breast cancer cell lines, MDA-MB-435, MDA-MB-468, SK-BR-3, and a normal cell line HEK-293, were grown to $80 \%$ confluency in T75 flasks and their RNA was isolated using Trizol (Ambion, Life Technologies, Grand Island, NY). Total RNA was quantified by spectrophotometry and $500 \mathrm{ng}$ was reverse transcribed to cDNA using SuperScript III Reverse Transcriptase (200 units $/ \mu \mathrm{L})$ and oligodT primers $(2.5 \mu \mathrm{M})$ (Life Technologies, Grand Island, NY). The subsequent cDNA was utilized for PCR reactions with egfl6 specific primers previously demonstrated to amplify the gene of interest [10]. The primers [(5'-CGGGATCCCTGTGCTACGTCGCCCTGGAC-3') and (5'-CGGAATTCACTGGCGCAGGCGGTGATCTCCTT-3')] were diluted to $10 \mu \mathrm{M}$ and added to $2 \mu \mathrm{L}$ of cDNA for the reaction. The cycling conditions were $98^{\circ} \mathrm{C}$ for $30 \mathrm{~s}$ for one cycle, followed by 30 cycles of $98^{\circ} \mathrm{C}$ for $10 \mathrm{~s}, 60^{\circ} \mathrm{C}$ for $30 \mathrm{~s}$ and $72^{\circ} \mathrm{C}$ for $30 \mathrm{~s}$. Primers for beta-actin were used as a loading control. PCR products were run on $1 \%$ agarose gels and visualized by ethidium bromide staining.

\section{Immunoassay}

Each cell line was analyzed by immunoassay for EGFL6 protein expression in the cell lysate and supernatant using a polyclonal antiEGFL6 antibody (Prestige Antibodies, Sigma, St. Louis, MO). Cells were grown to $80 \%$ confluency, supernatant harvested and both cells and supernatant were incubated with laemmli buffer ( $2 \% \mathrm{w}: \mathrm{v}$ sodium dodecyl sulfate, $10 \%$ glycerol, $60 \mathrm{mM}$ Tris, $0.01 \%$ bromophenol blue). Protein concentrations were determined by Bio-Rad protein assay (Bio-Rad, Hercules, CA) and $500 \mu \mathrm{g}$ of total protein was incubated with 
NuPAGE LDS sample buffer (Life Technologies, Grand Island, NY) at $80^{\circ} \mathrm{C}$ for $10 \mathrm{~min}$ and loaded onto NuPAGE Novex 4-12\% Bis-Tris gels. Samples were electrophoresed for $90 \mathrm{~min}$ at $120 \mathrm{mV}$ and transferred to $0.2 \mu \mathrm{m}$ nitrocellulose membrane (Bio-Rad, Hercules, CA). Following transfer, membranes were blocked with $5 \%$ non-fat dry milk. Blocked membranes were incubated with anti-EGFL6 antibody diluted 1:50 in Tris buffered saline and $0.1 \%$ Tween-20 (0.1\% TBST) for $10 \mathrm{~min}$ and vacuum aspirated by SNAP i.d. (Merck Millipore, Billerica, MA). Membranes were washed three times with $0.1 \%$ TBST and polyclonal anti-rabbit horseradish peroxidase-conjugated antibody diluted $1: 1000$ in $0.1 \%$ TBST was incubated with the membrane for $10 \mathrm{~min}$. Vacuum aspiration and washing were completed as with the primary antibody. Membranes were developed using SuperSignal West Pico chemiluminescent substrate (ThermoFisher Scientific, Rockford, IL) and visualized using a VersaDoc Molecular Imager (Bio-Rad, Hercules, CA).

\section{Peptide synthesis}

Synthesis of all peptides was accomplished with an Advanced Chem Tech 396 multiple peptide synthesizer using solid phase FMOC chemistry. Peptides were designed with an N-terminal GSG peptide spacer covalently linked at its $\mathrm{N}$-terminus with either biotin or 1,4,7,10-tetraazacyclododecane-1,4,7,10-tetraacetic acid (DOTA) (Macrocyclic, Inc. Dallas, TX).

\section{Biotinylated peptide fluorescent microscopy}

All cell lines were grown to $80 \%$ confluency and fixed with $4 \%$ paraformaldehyde. Cells were dried onto microscope slides overnight followed by rehydration with PBS. Slides were blocked with 5\% BSA in PBS for $2 \mathrm{~h}$ at room temperature. Biotinylated EGFL6 peptide was diluted to $10 \mu \mathrm{M}$ in PBS and $100 \mu \mathrm{L}$ was added to each cell sample for incubation at room temperature for $1 \mathrm{~h}$. Slides were washed 3 times with $0.1 \%$ TBST and cells were incubated with Neutravidin Texas Red (Life Technologies, Rockville, MD) diluted to $5 \mu \mathrm{g} / \mathrm{mL}$ in $0.1 \%$ TBST for $1 \mathrm{~h}$ at room temperature. Washing was performed in the same manner and cells were visualized by an epifluorescent Nikon T1-SM inverted microscope (Nikon, Melville, NY).

\section{DOTA-EGFL6 radiolabeling and purification}

DOTA-EGFL6 was diluted to $1 \mathrm{mg} / \mathrm{mL}$ in water and incubated with $0.1 \mathrm{M}$ ammonium acetate ( $\mathrm{pH}$ ) and $18.5 \mathrm{MBq}$ of ${ }^{111} \mathrm{In}$ at $80^{\circ} \mathrm{C}$ for 1 $\mathrm{h}$. Reactions were quenched by addition of $10 \mu \mathrm{M}$ EDTA. Radiolabeled peptide was purified by reverse phase HPLC using a linear gradient of acetonitrile plus $0.1 \%$ trifluoroacetic acid from 5 -95\% over 35 minutes. Acetonitrile was evaporated by nitrogen gas flow, and peptide was diluted to appropriate concentration using sterile PBS.

\section{${ }^{111}$ In-DOTA-EGFL6 cell binding}

MDA-MB-435, MDA-MB-468, SK-BR-3 and HEK-293 cells were diluted to $2 \times 10^{6}$ cells $/ \mathrm{mL}$ in Dulbelco's Modified Eagle Medium (DMEM) with $0.1 \mathrm{mg} / \mathrm{mL}$ BSA. ${ }^{111} \mathrm{In}-\mathrm{DOTA}-E G F L 6$ was diluted to $1 \times 10^{7} \mathrm{CPM} / \mathrm{mL}$ in DMEM plus $0.1 \% \mathrm{BSA}$ and $100 \mu \mathrm{L}$ of peptide was added to $200 \mu \mathrm{L}$ of cells and incubated at $37^{\circ} \mathrm{C}$ for $1 \mathrm{~h}$. Cells were washed three times with ice-cold PBS with $1 \%$ BSA and counted via gamma counter.

\section{${ }^{111}$ In-DOTA-EGFL6 Biodistribution and MicroSPECT/CT Imaging}

DOTA-EGFL6 peptide was radiolabeled with ${ }^{111}$ In and purified peptide was prepared at $1.85 \mathrm{MBq} / \mathrm{mL}$ in sterile PBS. Three mice bearing MDA-MB-435 tumors were intravenously injected with ${ }^{111} \mathrm{In}$ DOTA-EGFL6 and sacrificed at $2 \mathrm{~h}$. Organs and tissues pertinent to tumor uptake and clearance were excised, weighed, and counted by gamma counter. Percentage of injected dose per gram $(\% \mathrm{ID} / \mathrm{g})$ of tissue was reported to normalize uptake by tissue mass.

Radiolabeled, purified ${ }^{111}$ In-DOTA-GSG-EGFL6 was diluted to 11.1 $\mathrm{MBq}$ in $100 \mu \mathrm{L}$ of sterile PBS. The peptide was injected intravenously in a mouse bearing an MDA-MB-435 human breast cancer xenograft. Following injection, radiolabeled peptide was permitted to circulate for $2 \mathrm{~h}$, followed by euthanization by carbon dioxide. The treated mouse was imaged at the Harry S. Truman Veterans Memorial Hospital Biomolecular Imaging Center. Overnight (7h) image acquisition was performed with a Siemens Inveon Micro-SPECT/CT (Siemens, Knoxville, TN) equipped with mouse whole body $1.0 \mathrm{~mm}$ collimators. Data were processed with Inveon Research Workplace processing software and fan beam (Feldkamp) filtered back projection algorithms were used for reconstruction of the CT tomographic image.

\section{Results}

\section{Phage display selection and analysis}

Completion of four rounds of in vivo phage display selection resulted in a sub-population of presumed human breast tumor-avid phage clones. DNA sequence corresponding to the foreign displayed peptide of 269 tumor avid phages was obtained and analyzed by the BLAST algorithm for homology to human cancer-related proteins [23]. For each peptide sequence, the top 10 matching proteins were evaluated for percent homology and previous identification as a cancer-related or putative cancer-related protein. One displayed peptide, from the phage clone 3-G03, revealed 64\% identical homology to a protein termed EGFL6 (Figure 1). The match returned a score of 24.0 bits consisting of $9 / 14$ positive identities and 0 gaps. The match returned an expected value of 4.9, indicating that random assignment of amino acids would only return approximately 5 random matches in the entire protein database. No other sequence returned a cancer-related protein and had sequence similarity greater than $50 \%$.

\section{EGFL6 RT-PCR and immunoassay}

Due to the significant homology of the phage displayed peptide to EGFL6, it was inferred that the MDA-MB-435 cell line used for xenograft establishment for the in vivo phage display selection expressed EGFL6. In order to confirm egfl6 transcription, RT-PCR was performed. Additionally, two more breast cancer cell lines, MDAMB-468 and SK-BR-3, and a non-cancer cell line, HEK-293, were also assessed. PCR products from EGFL6 specific primers first used to identify the mRNA were visualized by ethidium bromide stain in agarose gel [10]. The results demonstrated a band corresponding to the expected fragment size of 801 nucleotides in the lane corresponding to

$\begin{array}{rcccc} & \mathbf{4 0} & \mathbf{5 0} & \mathbf{6 0} & \mathbf{7 0} \\ \text { EGFL6 } & \text { I } & \text { P GVCHYGTKLACCYGWRRNSKGVCEATCEPGCK } \\ \text { 3-G03 } & & \text { GTKSKCCYSLRRSS } & \mathbf{I}\end{array}$

Figure 1: The in vivo selected peptide 3-G03 was aligned with EGFL6 for sequence comparison. A portion of the full length EGFL 6 protein corresponding to the homologous selected peptide is displayed, with the hashed numbers representing the amino acid position from the $\mathrm{N}$-terminus. Identical matches are highlighted 
Citation: Larimer BM, Deutscher SL (2014) Identification of a Peptide from In vivo Bacteriophage Display with Homology to EGFL6: A Candidate Tumor Vasculature Ligand in Breast Cancer. J Mol Biomark Diagn 5: 178. doi:10.4172/2155-9929.1000178

MDA-MB-435 cDNA and a fainter band in the lane containing cDNA from MDA-MB-468 cells (Figure 2 and 3). No band was identified in the SK-BR-3 and HEK-293 lanes. Loading controls were accomplished using beta actin-specific primers producing a 315 nucleotide band, which was found in similar intensity in all cell lines analyzed.

Identification of egfl at the mRNA level provided impetus to analyze protein expression of each cell line. Since EGFL6 is a secreted protein, both the cells and the cultured supernatant were used for immunoblotting. A band was identified in the supernatant of MDAMB-435 cells that corresponded to the expected molecular weight of EGFL6, $66 \mathrm{kDa}$. No band was detected in the supernatant of all other cell lines, nor the cell pellet of any cells examined, including MDAMB-435.

\section{Biotinylated EGFL6 peptide fluorescent microscopy}

In order to confirm EGFL6 peptide affinity for MDA-MB-435 cells, the peptide sequence of EGFL6 (GTKWACCYGWRNSS) directly corresponding to the identified phage displayed peptide was synthesized and conjugated at the $\mathrm{N}$-terminus with biotin for detection by fluorophore labeled streptavidin. Peptide binding was analyzed in the same four cell lines, MDA-MB-435, MDA-MB-468, SK-BR-3 and HEK-293, used for RT-PCR and immunoassay experiments. The results demonstrated the peptide bound strongly to MDA-MB- 435 and MDA-MB-468 cells, while showing less binding to SK-BR-3 cells and no binding to HEK-293 cells (Figure 4).

\section{${ }^{111}$ In-DOTA-EGFL6 cell binding}

After confirmation of cell binding with biotinylated peptide, the peptide was conjugated to the macrocyclic chelator DOTA for radiolabeled peptide binding assays. Radiolabeled peptide was examined for its ability to bind MDA-MB-435, MDA-MB-468, SKBR-3 and HEK-293 cells. Peptide binding was determined to be 12.3 $\pm 1.0 \%$ (12284 CPM) of total peptide added for MDA-MB-435, $10.5 \%$ $\pm 2.7 \%$ (10508 CPM) for MDA-MB-468, $4.7 \pm 0.2 \%$ (4731 CPM) for SK-BR-3 and $5.4 \pm 0.5 \%$ (5411 CPM) for HEK-293 cells (Figure 5).

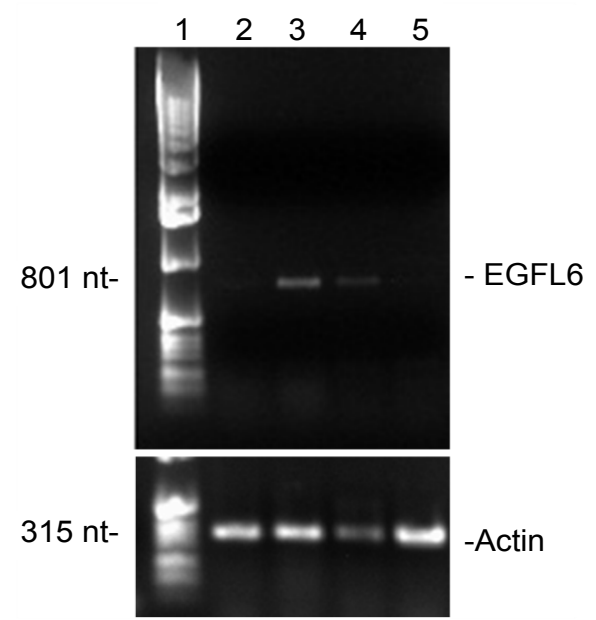

Figure 2: Isolated RNA from 3 human breast cancer cell lines and one human kidney cell line was reverse transcribed and amplified using EGFL6-specific primers. Bands of the expected nucleotide length were detected in MDAMB-435 and MDA-MB-468 samples. Additionally, a primer for $\beta$-Actin was used as a loading control. Numbered lanes represent the following: 1) Ladder, 2) SK-BR-3, 3) MDA-MB-435, 4) MDA-MB-468, 5) HEK-293.

\section{$\begin{array}{llllllllll}1 & 2 & 3 & 4 & 5 & 6 & 7 & 8 & 9 & 10\end{array}$

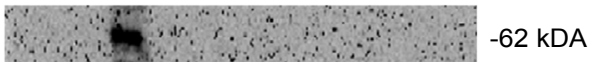

Figure 3: Total protein from cell lysates and cultured medium from corresponding cells were run on SDS-PAGE gels, transferred to nitrocellulose, and EGFL6 detected by immunoblot. One band was detected at the expected molecular weight for EGFL6 in the cultured supernatant of MDA-MB-435 cells Numbered lanes represent the following: 1) Ladder, 2) MDA-MB-435 cell lysate, 3) MDA-MB-435 supernatant, 4) MDA-MB-468 cell lysate, 5) MDAMB-468 supernatant, SK-BR-3 cell lysate, 6) SK-BR-3 supernatant, 7) HEK293 cell lysate, 8) HEK-293 supernatant, 9) ladder, 10) loading buffer.

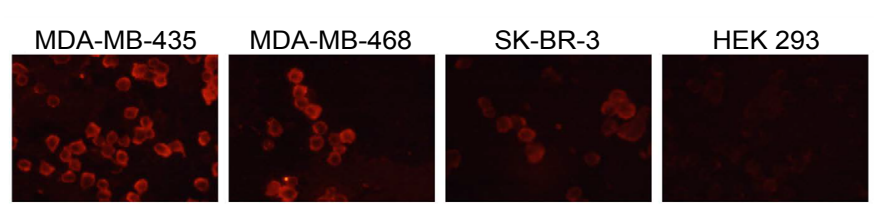

Figure 4: Human breast cancer and kidney cells were incubated with biotinylated peptides and visualized by fluorescent neutravidin. Fluorescent signal was detected in MDA-MB-435 and MDA-MB-468 cells, but not in SKBR-3 or HEK-293 cells.

The binding of ${ }^{111}$ In-DOTA-EGFL6 was significantly higher to MDAMB-435 than SK-BR-3 $(<0.001)$ and HEK-293 $(P<0.001)$ cells. Peptide binding was similar between MDA-MB-435 and MDA-468 cells and binding to MDA-MB-468 cells was also significantly higher $(P<0.05)$ than binding to SK-BR-3 or HEK-293 cells.

\section{${ }^{111}$ In-DOTA-EGFL6 biodistribution}

${ }^{111}$ In-DOTA-EGFL6 binding to MDA-MB-435 cells in vitro warranted in vivo analysis of the tumor targeting and non-target organ accumulation of EGFL6. In order to ascertain a preliminary understanding of the biodistribution, the pharmacokinetics of the peptide were analyzed at $2 \mathrm{~h}$ post-injection (Figure 6). Tumor uptake of the radiolabeled peptide was $0.36 \pm 0.08 \% \mathrm{ID} / \mathrm{g}$, while blood retention of the peptide was $1.30 \pm 0.51 \% \mathrm{ID} / \mathrm{g}$. Non-target organ accumulation was below $1.0 \% \mathrm{ID} / \mathrm{g}$ for all organs measured, except for the kidneys. Kidney retention of the peptide was $28.61 \pm 4.24 \% \mathrm{ID} / \mathrm{g}$. The tumor to blood ratio of the peptide was 0.27 , while the tumor to muscle ratio was 2.7.

\section{${ }^{111}$ In-DOTA-GSG-EGFL6 SPECT/CT imaging}

In addition to biodistribution, tumor imaging capabilities of ${ }^{111} \mathrm{In}$ DOTA-GSG-EGFL6 were explored. SPECT/CT images were collected at $2 \mathrm{~h}$ post-injection in order to correspond with the biodistribution data (Figure 7). Imaging revealed low tumor uptake, however, the interface between the xenograft and the muscle of the mouse had a strikingly high concentration of radiolabel. Kidney retention was also visible in the mouse, in addition to apparent cranio-facial, esophageal, and stomach intake, consistent with incidental ingestion of the radiolabeled peptide following oral cleaning of the injection site.

\section{Discussion}

Angiogenesis in cancer is a key component of tumor progression and, as such, has received much attention in both basic and translational research. Clinically-approved drugs including bevacizumab (Avastin ${ }^{\circ}$; Genentech) and sunitinib (Sutent ${ }^{\oplus}$; Sugen) in addition to counterparts in clinical trials, including VEGF-Trap ${ }_{\mathrm{R} 1 \mathrm{R} 2}$ (Aflibercept; Regeneron Inc.) and vendatanib (Caprelsa; AstraZeneca), illustrate the major emphasis 
Citation: Larimer BM, Deutscher SL (2014) Identification of a Peptide from In vivo Bacteriophage Display with Homology to EGFL6: A Candidate Tumor Vasculature Ligand in Breast Cancer. J Mol Biomark Diagn 5: 178. doi:10.4172/2155-9929.1000178

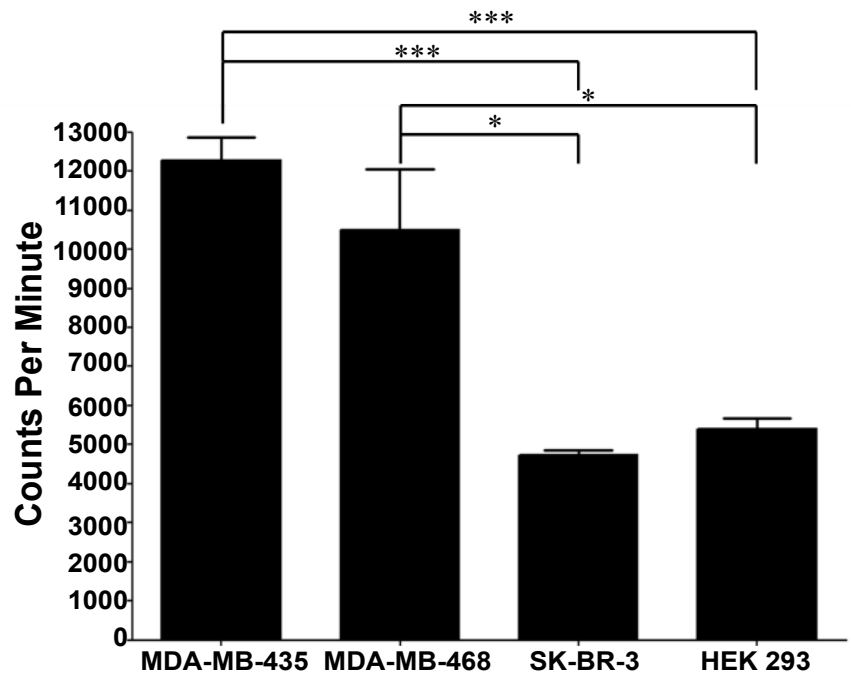

Figure 5: ${ }^{111}$ In-DOTA-EGFL6 peptide was incubated with cell lines and binding was quantified. The peptide bound to MDA-MB-435 and MDA-MB-468 cells significantly higher than SK-BR-3 and HEK-293 cells. Error bars represent the standard deviation of 4 samples. *- $P<0.05^{* * *}-P<0.001$.

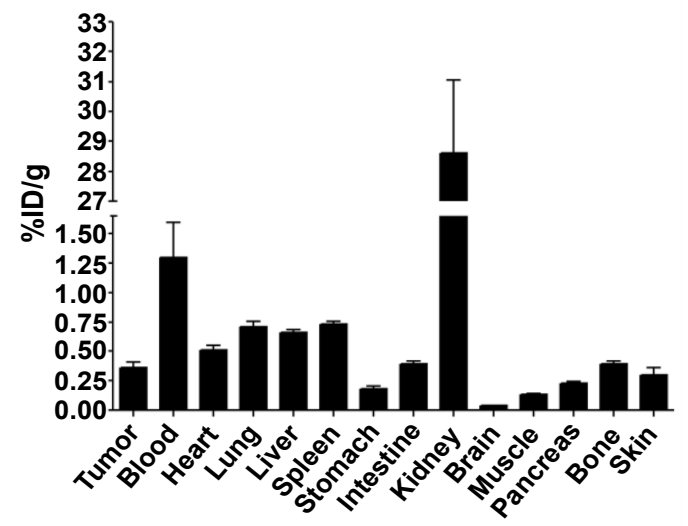

Figure 6: ${ }^{111} \mathrm{In}-\mathrm{DOTA}-E G F L 6$ was injected into mice bearing MDA-MB-435 human breast cancer xenografts. Tissue biodistribution was analyzed $2 \mathrm{~h}$ post injection. Bars represent the mean of $n=4$ and error bars signify the standard deviation. $\% \mathrm{ID} / \mathrm{g}$ - percent injected dose/gram.

placed on preventing tumor angiogenesis [24-27]. Unfortunately, response to current anti-angiogenic therapy is generally transient, and relapse is common [28]. One hypothesis for the eventual ineffectiveness of anti-angiogenic therapy is circumvention of therapeutic blockade by upregulation of complementary growth factors and receptors, such as EGFR and fibroblast Bv8 [29,30]. Identification of the potential ligands that promote tumor angiogenesis is necessary to help overcome resistance to current therapies. Although the list of tumor-associated ligands is extensive, it is unlikely to be complete [1].

Proteins involved in tumorigenesis can be identified at three levels: the genome, transcriptome and proteome. While high throughput DNA, RNA and protein expression interrogation techniques provide unique advantages including large sample size, quantification and breadth of results, each by itself is incomplete. Genetic differences may not be transcribed, transcriptional differences may not be translated, and proteomic differences may be too small to be distinguished.
Additionally, ex vivo analysis cannot directly investigate physiological location or function. In order to supplement these techniques, a method, such as in vivo phage display, can be used to explore protein interactions in a physiological environment. In vivo phage display has been utilized previously to map vascular signatures and identify novel tumor-associated proteins, such as proteome activator complex 28 and plectin-1 [20,31,32]. In vivo phage display provides two key supplementary features to current high throughput screening techniques: the ability to identify proteins that are expressed at low levels and not likely to be recognized by proteomic procedures, and the potential to identify targets that are biologically accessible for targeted imaging and therapy. Therefore, it was hypothesized that an in vivo phage display selection could identify tumor associated ligands by isolation of peptides with a similar sequence and function.

The in vivo selection enabled identification of peptides with tumor imaging capabilities in addition to providing an opportunity to explore whether peptides were similar to sequences of known or putative cancer proteins. Peptides corresponding to 267 phage clones recovered from human breast cancer xenografts were analyzed by the BLAST algorithm for homology to known cancer-related proteins. Since the sequences being analyzed were relatively short ( 15 amino acids), it was necessary to arbitrarily limit the scope of what was considered a match to greater than $50 \%$ homology. This greatly reduced the number of artificial hits that would be generated by random chance. Of the 267 peptides analyzed, only one, 3-G03, fit the criteria specified. 3-G03 (GTKSKCCYSLRRSS) matched a secreted protein, EGFL6 (GTKLACCYGWRRNS), with 64\% homology and returned a score of 24 bits, primarily due to the number of identical matches (9) and the

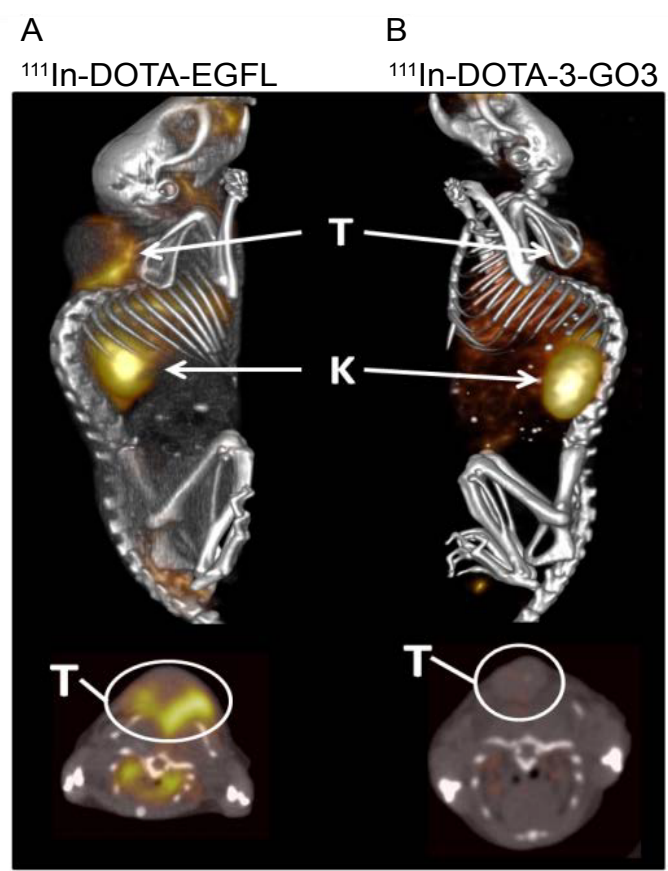

Figure 7: ${ }^{111}$ In-DOTA-EGFL6 and ${ }^{111}$ In-DOTA-3-G03 were injected into MDAMB-435 human breast carcinoma tumor bearing mice. SPECT/CT imaging was acquired $2 \mathrm{~h}$ post-injection. Uptake of ${ }^{111} \mathrm{In}$-DOTA-EGFL 6 is consistent with tumor vasculature accumulation. Sequence specificity of the EGFL6 peptide was confirmed by using the 3-G03 peptide, which differs by only 5 amino acids. 3-G03 did not have a tumor uptake pattern similar to EGFL6, but did show similar kidney uptake, which is typical of radiolabeled peptides. 
absence of gaps needed to make the identical pairs. It was interesting to note that the 9 identical pairs consisted of 2 tripeptide sequences, and an additional 4 peptide sequence with one mismatch. This is consistent with previous selections that have identified three amino acid sequences capable of mediating interaction between a ligand and a receptor, as is the case of RGD and several integrins [17]. Furthermore, tripeptide motifs have been demonstrated to target phage to specific organs in vivo, including the tumor vasculature [20]. The high homology between 3-G03 and EGFL6, in addition to the potential tumor vasculature promoting properties of the protein, led to further investigation into EGFL6 expression and peptide characterization.

Because a peptide with homology to EGFL6 was identified by the in vivo selection, it was assumed that the xenografted breast cancer cell line expressed EGFL6. For confirmation, RT-PCR was used to analyze egfl6 transcription in three breast cancer cell lines and a control cell line. The results confirmed that egfl 6 was expressed in MDA-MB- 435 cells, in addition to MDA-MB-468 cells, although at apparently lower levels. This result was consistent with identification of egfl6 expression in biopsy samples from ovarian and breast cancer, as well as meningioma $[6,8,13]$.

While eglf6 mRNA has been identified, EGFL6 protein has not been confirmed in cultured carcinomas. In order to verify the egfl6 transcript was translated, an immunoassay probing EGFL6 expression was performed. EGFL6 is a secreted protein; therefore immunoassay was performed on both the cultured supernatant and cell pellet of all cell lines previously analyzed. Interestingly, EGFL6 protein was only identified in the supernatant of MDA-MB- 435 cells and not in MDAMB-468 cells. Lack of detectable protein in MDA-MB-468 cells could have resulted from the protein concentration being undetectable by immunoassay, which is feasible due to the apparent diminished amount of mRNA detected by RT-PCR. Also EGFL6 translation in MDAMB-468 cells may have been disrupted, as protein production in cancer cells has been demonstrated to be aberrant $[33,34]$. Additionally, the protein may be degraded rapidly under in vitro conditions by the cells. Detection of EGFL6 protein expression in cultured MDA-MB-435 cells confirmed the assumption that EGFL6 was expressed by the cell line used in the in vivo selection and provided motivation to further investigate the properties of the EGFL6 peptide.

Although EGFL6 protein expression was demonstrated by immunoblot, the affinity and specificity of the corresponding EGFL6 peptide for human breast carcinomas needed to be assessed. The exact peptide sequence for EGFL6 corresponding to the phage displayed homologue was selected for analysis. This approach was chosen to maximize the possibility that the properties of the peptide were mediated by the natural EGFL6 sequence, and not selected by phage display. EGFL6 peptide was chemically synthesized with a covalently linked biotin to determine in vitro peptide specificity for the cell lines previously analyzed. Fluorescent microscopy revealed strong binding to MDA-MB-435 and MDA-MB-468 human breast cancer cells. Binding was undetectable to SK-BR-3, a third human breast cancer cell line, and HEK-293 human kidney cells. Peptide affinity for the carcinomas corresponded with cell lines that expressed egfl6 mRNA, even though the protein expression would suggest binding in only MDA-MB-435 cells. The receptor for EGFL6 is not known; therefore it is impossible to predict how the peptide or protein may bind to cells. A correlation between peptide binding and egfl 6 mRNA expression suggests that peptide uptake may predict cellular expression of the egfl6 transcript. Obviously a short peptide sequence cannot fully mimic a full-length protein, which may contain several domains, post-translational modification sites and tertiary structure $[10,14]$.

Identification of a peptide that bound to human breast carcinoma cells expressing EGFL6 offered the potential that the EGFL6 peptide could be used in vivo to detect tumorigenesis in xenografts expressing the potential tumor vasculature ligand. To evaluate the biodistribution and imaging properties of the EGFL6 peptide, it was necessary to conjugate the peptide to a radiometal chelator. Bifunctional macrocyclic DOTA was chosen due to its stable chelation with a number of radiometals, including indium-111, and well understood in vivo properties [35-37]. Prior to in vivo studies, retained specificity and affinity of the radiolabeled peptide was monitored by in vitro cell binding. ${ }^{111} \mathrm{In}$-DOTA-EGFL6 bound to MDA-MB-435 and MDAMB-468 cells significantly higher than SK-BR-3 or HEK-293 cells, confirming retention of the breast cancer targeting properties of the peptide.

Radiolabeled, purified ${ }^{111}$ In-DOTA-EGFL6 was injected into mice bearing MDA-MB-435 human breast cancer xenografts for biodistribution studies. Surprisingly, tumor uptake was low at $0.36 \pm$ $0.08 \% \mathrm{ID} / \mathrm{g}$. The tumor to blood ratio was also suboptimal at 0.27 , but a tumor to muscle ratio of 2.7 indicated specificity for the tumor. A corresponding SPECT/CT image was acquired for further insight into the low tumor uptake. Image analysis correlated with the results of the biodistribution. Although the solid tumor lacked significant uptake of ${ }^{111}$ In-DOTA-EGFL6, there appeared to be a high concentration at the interface of the tumor and the site of xenograft formation, presumably a sight of high neovascularization [38]. The SPECT/CT image was consistent with other vasculature-targeted imaging agents, such as radiolabeled RGD peptide [39]. Absence of solid tumor-retention could be the result of peptide binding to its target at the novel vasculature and not permitting further diffusion into the tumor [40]. Another reason for low tumor uptake might be differential expression of the receptor for EGFL6 in vitro and in vivo. In vivo expression of the receptor may be limited to cells near the site of neovascularization, thus limiting uptake to the interface of tumor and normal endothelial cells [40]. Previous work had demonstrated the phage selected peptide, 3-G03, bound to MDA-MB-435 cells in vitro, and its sequence similarity to EGFL6 made ${ }^{111}$ In-DOTA-3-G03 a unique control to determine whether the precise EGFL6 sequence was necessary for its in vivo imaging properties. While a very slight tumor uptake was observed for ${ }^{111}$ In-DOTA-3-G03, the distinct vasculature-like binding observed with ${ }^{111}$ In-DOTA-EGFL6 was not present in the ${ }^{111}$ In-DOTA-3-G03 images. Kidney uptake, which is not sequence specific and instead a result of filtration of low molecular weight peptides, was present for both peptides. SPECT/ CT imaging revealed that EGFL6 was targeted to the tumor-epithelial interface of human breast carcinoma xenografts, and the binding was specific to the EGFL6 peptide sequence.

\section{Conclusion}

An in vivo phage display selection resulted in the identification of a potential tumor vasculature ligand, EGFL6. Additionally, a peptide with homology to EGFL6 was radiolabeled with ${ }^{111}$ In and used to image tumor vasculature by SPECT/CT. This data presents evidence that EGFL6 should be further investigated for its roles in tumorigenesis and as a possible imaging agent.

\section{Acknowledgements}

The authors would like to acknowledge the contributions of Jessica NewtonNorthup, Marie T. Dickerson and the VA Biomolecular Imaging Core. This 
Citation: Larimer BM, Deutscher SL (2014) Identification of a Peptide from In vivo Bacteriophage Display with Homology to EGFL6: A Candidate Tumor Vasculature Ligand in Breast Cancer. J Mol Biomark Diagn 5: 178. doi:10.4172/2155-9929.1000178

material is based upon work supported (or supported in part) by the Department of Veterans Affairs, Veterans Health Administration, Office of Research and Development, Biomedical Laboratory Research and Development, Clinical Sciences Research and Development including the Cooperative Studies Program, Rehabilitation Research and Development Service, and Health Services Research and Development through a VA Merit Award (I01BX000964). The work is also supported by an NIH R21 grant (1R21CA179069-01).

\section{References}

1. Shojaei $F$ (2012) Anti-angiogenesis therapy in cancer: current challenges and future perspectives. Cancer Lett 320: 130-137

2. Folkman J (1971) Tumor angiogenesis: therapeutic implications. N Engl J Med 285: 1182-1186.

3. Ruoslahti E, Rajotte D (2000) An address system in the vasculature of normal tissues and tumors. Annu Rev Immunol 18: 813-827.

4. Jain RK (1997) The Eugene M. Landis Award Lecture 1996. Delivery of molecular and cellular medicine to solid tumors. Microcirculation 4: 1-23.

5. Weis SM, Cheresh DA (2011) Tumor angiogenesis: molecular pathways and therapeutic targets. Nat Med 17: 1359-1370.

6. Sasaroli D, Gimotty PA, Pathak HB, Hammond R, Kougioumtzidou E, et al. (2011) Novel surface targets and serum biomarkers from the ovarian cancer vasculature. Cancer Biol Ther 12: 169-180.

7. DeRisi J, Penland L, Brown PO, Bittner ML, Meltzer PS, et al. (1996) Use of cDNA microarray to analyse gene expression patterns in human cancer. Nat Genet 14: 457-460.

8. Li JL, Harris AL (2007) The potential of new tumor endothelium-specific markers for the development of antivascular therapy. Cancer Cell 11: 478-481.

9. Bild AH, Yao G, Chang JT, Wang Q, Potti A, et al. (2006) Oncogenic pathway signatures in human cancers as a guide to targeted therapies. Nature 439: 353-357.

10. Yeung G, Mulero JJ, Berntsen RP, Loeb DB, Drmanac R, et al. (1999) Cloning of a novel epidermal growth factor repeat containing gene EGFL6: expressed in tumor and fetal tissues. Genomics 62: 304-307.

11. Buckanovich RJ, Sasaroli D, O'Brien-Jenkins A, Botbyl J, Hammond R, et al (2007) Tumor vascular proteins as biomarkers in ovarian cancer. J Clin Oncol 25: 852-861.

12. Poola I, Abraham J, Marshalleck JJ, Yue Q, Fu SW, et al. (2009) Molecular constitution of breast but not other reproductive tissues is rich in growth promoting molecules: a possible link to highest incidence of tumor growths. FEBS Lett 583: 3069-3075.

13. Wang X, Gong Y, Wang D, Xie Q, Zheng M, et al. (2012) Analysis of gene expression profiling in meningioma: deregulated signaling pathways associated with meningioma and EGFL6 overexpression in benign meningioma tissue and serum. PLoS One 7: e52707.

14. Chim SM, Qin A, Tickner J, Pavlos N, Davey T, et al. (2011) EGFL6 promotes endothelial cell migration and angiogenesis through the activation of extracellular signal-regulated kinase. J Biol Chem 286: 22035-22046.

15. Smith GP (1985) Filamentous fusion phage: novel expression vectors that display cloned antigens on the virion surface. Science 228: 1315-1317.

16. Ruoslahti E (2000) Targeting tumor vasculature with homing peptides from phage display. Semin Cancer Biol 10: 435-442

17. Koivunen E, Gay DA, Ruoslahti E (1993) Selection of peptides binding to the alpha 5 beta 1 integrin from phage display library. J Biol Chem 268: 2020520210.

18. Ruoslahti E (1996) RGD and other recognition sequences for integrins. Annu Rev Cell Dev Biol 12: 697-715.

19. Jung E, Lee NK, Kang SK, Choi SH, Kim D, et al. (2012) Identification of tissuespecific targeting peptide. J Comput Aided Mol Des 26: 1267-1275.
20. Arap W, Kolonin MG, Trepel M, Lahdenranta J, Cardó-Vila M, et al. (2002) Steps toward mapping the human vasculature by phage display. Nat Med 8 : 121-127.

21. Newton JR, Kelly KA, Mahmood U, Weissleder R, Deutscher SL (2006) In vivo selection of phage for the optical imaging of PC-3 human prostate carcinoma in mice. Neoplasia 8: 772-780.

22. http://www.biosci.missouri.edu/smithGP/PhageDisplayWebsite/PhageDisplayWebsitelndex.htm

23. Altschul SF, Gish W, Miller W, Myers EW, Lipman DJ (1990) Basic local alignment search tool. J Mol Biol 215: 403-410.

24. Presta LG, Chen H, O'Connor SJ, Chisholm V, Meng YG, et al. (1997) Humanization of an anti-vascular endothelial growth factor monoclonal antibody for the therapy of solid tumors and other disorders. Cancer Res 57: 4593-4599.

25. Holash J, Davis S, Papadopoulos N, Croll SD, Ho L, et al. (2002) VEGF-Trap: a VEGF blocker with potent antitumor effects. Proc Natl Acad Sci U S A 99: 11393-11398.

26. O'Farrell AM, Abrams TJ, Yuen HA, Ngai TJ, Louie SG, et al. (2003) SU11248 is a novel FLT3 tyrosine kinase inhibitor with potent activity in vitro and in vivo. Blood 101: 3597-3605.

27. Langmuir PB, Yver A (2012) Vandetanib for the treatment of thyroid cancer Clin Pharmacol Ther 91: 71-80.

28. Kerbel RS (2008) Tumor angiogenesis. N Engl J Med 358: 2039-2049.

29. Cascone T, Herynk MH, Xu L, Du Z, Kadara H, et al. (2011) Upregulated stromal EGFR and vascular remodeling in mouse xenograft models of angiogenesis inhibitor-resistant human lung adenocarcinoma. J Clin Invest 121: 1313-1328.

30. Shojaei F, Wu X, Malik AK, Zhong C, Baldwin ME, et al. (2007) Tumor refractoriness to anti-VEGF treatment is mediated by $C D 11 b+G r 1+$ myeloid cells. Nat Biotechnol 25: 911-920.

31. Sánchez-Martin D, Torrecuadrada JM, Teesaluet T, Sugahara KN, Cienfuegos AA, et al. (2013) Proteasome activator complex PA28 identified as an accessible target in prostate cancer by in vivo selection of human antibodies. Proc Natl Acad Sci U S A 110: 13791-13796.

32. Bausch D, Thomas S, Mino-Kenudson M, Fernández-del CC, Bauer TW, et al (2011) Plectin-1 as a novel biomarker for pancreatic cancer. Clin Cancer Res 17: 302-309.

33. Bjornsti MA, Houghton PJ (2004) Lost in translation: dysregulation of capdependent translation and cancer. Cancer Cell 5: 519-523.

34. Koumenis C, Naczki C, Koritzinsky M, Rastani S, Diehl A, et al. (2002) Regulation of protein synthesis by hypoxia via activation of the endoplasmic reticulum kinase PERK and phosphorylation of the translation initiation factor elF2alpha. Mol Cell Biol 22: 7405-7416.

35. Chen J, Cheng Z, Miao Y, Jurisson SS, Quinn TP (2002) Alpha-melanocytestimulating hormone peptide analogs labeled with technetium-99m and indium-111 for malignant melanoma targeting. Cancer 94: 1196-1201.

36. Kumar SR, Deutscher SL (2008) 111 In-labeled galectin-3-targeting peptide as a SPECT agent for imaging breast tumors. J Nucl Med 49: 796-803.

37. Deutscher SL, Figueroa SD, Kumar SR (2009) 111In-labeled KCCYSL peptide as an imaging probe for ErbB-2-expressing ovarian carcinomas. J Labelled Comp Radiopharm 52: 583-590.

38. Carmeliet P, Jain RK (2000) Angiogenesis in cancer and other diseases Nature 407: 249-257.

39. Haubner R, Beer AJ, Wang H, Chen X (2010) Positron emission tomography tracers for imaging angiogenesis. Eur J Nucl Med Mol Imaging 37 Suppl 1 : s86-103.

40. McDonald DM, Choyke PL (2003) Imaging of angiogenesis: from microscope to clinic. Nat Med 9: 713-725 